\title{
A new mechanism for soft X-ray excess in NGC 5548
}

\author{
Tong-Sheng Xia and Jia-Lü Zhang \\ 1 Center for Astrophysics, University of Science and Technology of China, Hefei, 230026 Anhui, PR China \\ 2 National Astronomical Observatories, Chinese Acadamy of Sciences (NAOCAS), 100012 Beijing, PR China
}

Received 20 November 2000 / Accepted 12 January 2001

\begin{abstract}
Many active galaxies exhibit soft X-ray excess in the $0.2-2 \mathrm{keV}$ band with a photon index $\alpha_{\mathrm{s}} \sim$ $2.38 \pm 0.25$, much higher than that of the hard X-ray $(2-10 \mathrm{keV})$ band with $\alpha_{\mathrm{h}} \sim 1.7$. Different theoretical models have been proposed to reproduce and explain the spectra, but the complexity of all these models tends to make the situation even more confused. Here we present results using our newly-proposed synchro-curvature mechanism, which we show can reproduce the soft X-ray excess by a simple fitting process. Such a model may be helpful in considering the physical processes taking place in these objects.
\end{abstract}

Key words. galaxies: individual: NGC 5548 - radiation mechanisms: non-thermal - magnetic fields - X-rays: galaxies

\section{Introduction}

Various classes of active galaxies have been studied in the $2-10 \mathrm{keV} \mathrm{X}$-ray band over the last 20 years. From HEAO 1 observations in the early 1980s, the spectra in this band can be well approximated by a simple powerlaw model with the photon index tightly clustered around the "canonical" value of $\alpha_{\mathrm{h}} \sim 1.7$ (Mushotzky 1984). This was confirmed by numerous observations carried out using the medium-energy (ME) array on board EXOSAT.

In the mid-1980s, several observations using the Imaging Proportional Counter (IPC) on board the Einstein observatory revealed that the photon indexes of soft X-ray (0.1-2 keV) spectra from a number of individual galaxies can be significantly steeper than $\alpha_{\mathrm{h}} \sim 1.7$ (Pravdo \& Marshall 1984; Elvis et al. 1985). By a combined analysis of data from the EXOSAT ME and low-energy (LE) Experiments, a clear spectral steepening between the soft and hard X-ray bands, or so called "soft X-ray excess", was demonstrated in a single object, MKN 841 (Arnaud et al. 1985). From then on, numerous observations, especially those by IUE, ROSAT, Ginga and ASCA, focused on this X-ray band (Walter et al. 1994; Turner et al. 1993; George et al. 1998). However, the spectrum in the soft $\mathrm{X}$-ray band is still not as well characterised as that in the $2-10 \mathrm{keV}$ X-ray band. It is thought that the continuum may have an index $\alpha_{\mathrm{s}} \sim 2.38 \pm 0.25$ (Turner et al. 1993; Walter \& Fink 1993), especially in the Seyfert type AGNs.

Send offprint requests to: J.-L. Zhang,

e-mail: jlz@ustc.edu.cn
Understanding soft X-ray emission is also relevant in many related problems. For example, we believe that the X-ray emission originates in the inner portion of the AGNs. Thus, soft X-ray emission becomes crucial in order to understand processes occurring therein (Marshall et al. 1981). For the Seyfert type AGNs, the soft X-ray band is affected by the X-ray background (Elvis et al. 1978; Barcons \& Fabian 1992), which may limit their detection. It may also be important to separate the signal of the Big Blue Bump (BBB), which located close to the soft X-ray excess, from the underlying soft X-ray continuum. Other problems, such as the inner emissions in this band, the relative significance of Galactic absorption and the intrinsic absorption, and the inner zone structure of accretion disk, all affect the soft excess spectra.

The soft X-ray spectrum with an energy excess can be roughly approximated by models with a second powerlaw component (Turner et al. 1993; Turner \& Pounds 1989), or a thermal model such as a thin disk with thermal bremsstrahlung or a black body component (Arnaud et al. 1985). It has been suggested that the soft X-ray excess is the tail of the BBB (Czerny \& Elvis 1987), but this has been contradicted by the results of Laor et al. (1997) and Zheng et al. (1997). They found that the soft X-ray is more likely the extension of the UV band, and so the BBB may be less important than previously thought. Now much attention has been given to "reprocessing" models, in which the soft X-rays are thought of as being "secondary" emission produced by passive re-radiation of "primary" hard $\mathrm{X}$-ray photons in e.g., an accretion disk. However, recent observations showing no time lag between soft and hard 
X-ray bands may rule out the application of this process (Edelson et al. 2000).

In this paper we have applied our newly-proposed mechanism (Zhang \& Cheng 1995; Cheng \& Zhang 1996; Zhang et al. 2000) to this problem. Our "synchro-curvture mechanism" allows us to derive a spectrum with energy excess in the lower energy band, which we think may address the soft X-ray excess problem in AGNs. In previous models, assuming a power law spectrum with $\alpha_{\mathrm{x}} \sim 1.7$ throughout the X-ray band and then adding another component in the soft X-ray band, the two bands was considered separately. In our model, we use only one power-law component, as opposed to two different components, to explain the overall $0.2-10 \mathrm{keV}$ spectrum. The simplicity of our method makes it well-suited to an analysis of this intriguing problem.

Rather than focusing on solving this problem in its entirety, we hope to introduce the analytical mechanism itself and discuss its usefulness in considering similar questions. In support of its effectiveness, we note that if the interpretation proposed here is true, and the same electron population is responsible for the overall spectral shape, then one would expect the soft and hard X-ray emission to vary simultaneously, and the variability amplitude to be similar in both spectra. This prediction may be supported by the observe of no time lag between soft and hard X-ray spectra in recent observations by Edelson et al. (2000).

The synchro-curvature mechanism is introduced in Sect. 2. In Sect. 3, useful considerations on the application of the parameters are given. In Sect. 4, we study NGC 5548, a special source, applying our method and comparing its implications to those of previous studies. Conclusions on these results are presented in Sect. 5 .

\section{Synchro-curvature mechanism}

The power per unit frequency of synchro-curvature radiation in two polarizations is given by (Zhang \& Cheng 1995; Cheng \& Zhang 1996; Zhang et al. 2000):

$$
\begin{aligned}
\frac{\mathrm{d} P_{\perp}}{\mathrm{d} \omega}= & -\frac{\sqrt{3}\left[\left(r_{\mathrm{B}}+\rho\right) \Omega_{0}^{2}+r_{\mathrm{B}} \omega_{\mathrm{B}}^{2}\right]^{2} \gamma}{2 \pi 2 c^{4} Q_{2}^{2} r_{\mathrm{c}}} \\
& \times\left\{\frac{\omega}{\omega_{\mathrm{c}}}\left[K_{2 / 3}\left(\frac{\omega}{\omega_{\mathrm{c}}}\right)+\int_{\frac{\omega}{\omega_{\mathrm{c}}}}^{\infty} K_{5 / 3}(y) \mathrm{d} y\right]\right\}, \\
\frac{\mathrm{d} P_{\|}}{\mathrm{d} \omega}= & -\frac{\sqrt{3}}{2} \frac{e^{2} \gamma}{2 \pi} \frac{1}{r_{\mathrm{c}}} \\
& \times\left\{\frac{\omega}{\omega_{\mathrm{c}}}\left[\int_{\frac{\omega}{\omega_{\mathrm{c}}}}^{\infty} K_{5 / 3}(y) \mathrm{d} y-K_{2 / 3}\left(\frac{\omega}{\omega_{\mathrm{c}}}\right)\right]\right\},
\end{aligned}
$$

and

$\frac{\mathrm{d} P}{\mathrm{~d} \omega}=\frac{\mathrm{d} P_{\|}}{\mathrm{d} \omega}+\frac{\mathrm{d} P_{\perp}}{\mathrm{d} \omega}$

where the cyclotron frequency $\omega_{\mathrm{B}}=e B /(\gamma m c)$, with $\gamma$ the Lorentz factor of a relativistic electron, $m$ the electron mass, $e$ the electron charge, $c$ the velocity of light, $B$ the magnetic field, the cyclotron radius $r_{\mathrm{B}}=c \sin \alpha / \omega_{\mathrm{B}}$, with $\alpha$ the pitch angle, $\omega_{\mathrm{c}}$ is the characteristic frequency, $\Omega_{0}$ is the angular velocity of the guiding center along the magnetic field line, $\rho$ and $r_{\mathrm{c}}$ are the curvature radius of the magnetic field line and the particle trajectory, respectively. Also,

$$
\begin{aligned}
\omega_{\mathrm{c}}= & \frac{3}{2} \gamma^{3} c Q_{2} \equiv \frac{3}{2} \gamma^{3} c \frac{1}{\rho}\left[\frac{3 \rho}{r_{\mathrm{B}}} \cos ^{2} \alpha+\frac{\rho^{2}}{r_{\mathrm{B}}^{2}} \sin ^{4} \alpha\right. \\
& \left.+\frac{\left(r_{\mathrm{B}}^{3}+\rho r_{\mathrm{B}}^{2}-3 r_{\mathrm{B}} \rho^{2}\right)}{\rho r_{\mathrm{B}}^{2}} \cos ^{4} \alpha\right]^{1 / 2}
\end{aligned}
$$

and

$r_{\mathrm{c}}=\frac{c^{2}}{\left(r_{\mathrm{B}}+\rho\right) \Omega_{0}^{2}+r_{\mathrm{B}} \omega_{\mathrm{B}}^{2}}$

It can be shown that from the above formulae (1-4) we can deduce all the results of the synchrotron and curvature mechanisms under two extreme situations. However, for the synchro-curvature mechanism, the relation between $\mathrm{d} P / \mathrm{d} \omega$ and $\gamma$ is more complex. $\mathrm{d} P / \mathrm{d} \omega$ changes with $\gamma$ not only by $\omega_{\mathrm{c}}$, but also by $r_{\mathrm{c}}$ (i.e. it is no longer a simply power law function of $\gamma$ ). We can write:

$$
\begin{aligned}
\frac{1}{r_{\mathrm{c}}} & =\frac{m c^{2} \sin \alpha \cos ^{2} \alpha}{e B \rho^{2}} \gamma+\frac{\cos ^{2} \alpha}{\rho}+\frac{e B \sin \alpha}{m c^{2} \gamma} \\
& \equiv a_{0} \gamma+a_{1}+\frac{a_{2}}{\gamma} .
\end{aligned}
$$

When electrons have a power-law distribution: $N(\gamma)=$ $N_{0} \gamma^{-n}$, the resulting radiation spectrum in two polarizations is as follows:

$$
\begin{aligned}
J_{\perp}(\omega)= & -\frac{\sqrt{3} e^{2}}{4 \pi} \frac{9 \mathrm{c}^{2}}{4} \int_{\gamma_{1}}^{\gamma_{2}} N_{0} \gamma^{-n+7} \frac{1}{\omega_{\mathrm{c}}^{2}} \\
& \times\left\{\frac{\omega}{\omega_{\mathrm{c}}}\left[\int_{\frac{\omega}{\omega_{\mathrm{c}}}}^{\infty} K_{5 / 3}(y) \mathrm{d} y+K_{2 / 3}\left(\frac{\omega}{\omega_{\mathrm{c}}}\right)\right]\right\} \\
& \times\left[a_{0} \gamma+a_{1}+\frac{a_{2}}{\gamma}\right]^{3} \mathrm{~d} \gamma, \\
J_{\|}(\omega)= & -\frac{\sqrt{3} e^{2}}{4 \pi} \int_{\gamma_{1}}^{\gamma_{2}} N_{0} \gamma^{-n+1} \\
& \times\left\{\frac{\omega}{\omega_{\mathrm{c}}}\left[\int_{\frac{\omega}{\omega_{c}}}^{\infty} K_{5 / 3}(y) \mathrm{d} y-K_{2 / 3}\left(\frac{\omega}{\omega_{\mathrm{c}}}\right)\right]\right\} \\
& \times\left[a_{0} \gamma+a_{1}+\frac{a_{2}}{\gamma}\right] \mathrm{d} \gamma,
\end{aligned}
$$

and

$J(\omega)=J_{\|}(\omega)+J_{\perp}(\omega)$.

We can see from the above equations that the electrons' resulting radiation spectrum is the addition of different power-law radiation spectra. Thus, the synchro-curvature spectrum can naturally has a turnoff point or an energy excess in the lower frequency band (Zhang et al. 2000). 


\section{Considerations for applications}

To obtain the values of parameters, we use previously published values, and adjust other parameters consistent with the characteristics of the turnoff point and the physical conditions. For example, $\gamma$ should be limited. Usually, we use $\gamma_{\max } / \gamma_{\min }=10^{2}$, and $\gamma_{\max } / \gamma_{\mathrm{c}}=10$. Thus, changing $B, \rho$, or $\alpha$ can affect the shape of spectra in their main frequency region, which may be useful for modelling purposes and may help explain observations, such as an excess of soft X-ray (Rush \& Malkan 1996).

Different sources are constrained by different physical limitations, which should be taken into account in a model. For example, for neutron stars, the value of the magnetic field and the curvature of the magnetic field lines have been determined in the radiation region, and should be used as such. In the case of AGNs, we assign a typical value for the magnetic field, and, from observation, include the indexes of the higher frequency $\left(\alpha_{\mathrm{h}}\right)$ and the lower frequency $\left(\alpha_{\mathrm{s}}\right)$, we can then apply the following equations to estimate the electron energy distribution crudely:

$$
\begin{aligned}
& n=\frac{n_{1}+n_{2}+n_{3}}{3}, \\
& \frac{n_{1}-1}{2}=\alpha_{\mathrm{s}}, \frac{n_{2}-2}{3}=\alpha_{\mathrm{h}}, \\
& \frac{n_{3}-1}{2}-\frac{n_{3}-2}{3}=\alpha_{\mathrm{s}}-\alpha_{\mathrm{h}},
\end{aligned}
$$

in which $\frac{n-1}{2}$ is a synchrotron-like index, and $\frac{n-2}{3}$ is a curvature-like index.

The following two approximations are useful in determining the value of $\gamma_{\mathrm{c}}, \rho$, and $\alpha_{\mathrm{c}}$. Firstly, to produce a curved spectrum, we should have $a_{0} \gamma_{\mathrm{c}}=a_{2} / \gamma_{\mathrm{c}}$, resulting in

$$
e B \rho \simeq \gamma_{\mathrm{c}} m c^{2} \cos \alpha
$$

Secondly, $\omega_{\mathrm{c}}$ can be approximated as:

$\omega_{\mathrm{c}} \simeq \frac{3 c}{2 \rho} \gamma_{\mathrm{c}}^{3}$.

For sources like neutron stars, the curvature of the magnetic field lines in the radiation region have been determined. Using Eqs. $(10,11)$, we can approximate the magnitude of $\alpha_{\mathrm{c}}$ and $\gamma_{\mathrm{c}}$. If we make the range of $\alpha$ variable and adjust its value to fit observation, we arrive at the best fitting result of $\alpha_{\mathrm{c}}$. We then integrate $\alpha$ in its range with $\alpha_{\mathrm{c}}$ as the median, leaving us with only one variable parameter.

For sources whose magnetic field's curvature has not been determined, we can allow it to vary, while the other parameters are fixed, again resulting in one variable, $\rho$. Thus, our method reduces a complex system to only a single variable, showing the power of the synchro-curvature mechanism relative to other more complex models.

The following section discusses an application of this model to the source NGC 5548, which shows a soft X-ray excess.

\section{Application of model to NGC 5548}

Generally, there are two ways to model the energy distribution spectrum of an active galaxy. One is to determine the statistical properties of an ensemble of objects, and derive an "average AGN spectrum". Another approach is to model the spectrum of a well-observed AGN. In this paper, we prefer the second approach, i.e., to study the well-observed source NGC 5548, because more precise and complete observation data are available, improving the reliability of the parameters used. NGC 5548 is one of the brightest and variable Seyfert 1 AGNs, and has been observed since the 1970s. It became an intensively-monitored source in the 1980s during the ground-based ultraviolet (IUE) campaign ("AGN watch") by an international team of astronomers. Of course, intensive interest has also been focused on the X-ray band (Turner \& Pounds 1989; Walter et al. 1994; Done \& Kroloik 1995).

However, we still lack an overall SED (spectrum energy distribution). We do not know exactly the continuum shape in the hard X-ray and gamma-ray range, or even the EUV luminosity. Here, we focus our model just on the $0.2-10 \mathrm{keV}$ bands. Laor et al. (1997) and Zheng et al. (1997) proposed a very attractive idea: perhaps the soft $\mathrm{X}$-ray is just the extension of the UV band. This means the BBB may be less important than previously thought. The non-thermal origin of the soft X-ray excess may thus be more significant compared to the thermal origin. As we will show below, a single power law model may suffice. Assuming electrons having a power law energy distribution, the theoretical resulting spectrum can be enough to explain the soft X-ray excess. Our data for the $0.2-2 \mathrm{keV}$ band are taken from Walter et al. (1994) from IUE and ROSAT observations, and the $2-10 \mathrm{keV}$ band are from EXOSAT observations (Turner \& Pounds 1989).

We now apply our synchro-curvature mechanism to model the behaviour of NGC 5548. If we take $\alpha_{\mathrm{s}}=2.38$, $\alpha_{\mathrm{h}}=1.7$, using Eq. (9), then $n \simeq 4.2$. For $B$, we assign a typical value of $1 \mathrm{G}$, as before. Using Eqs. (10, 11), $\gamma_{\mathrm{c}}=10^{5}, \gamma_{\max }=10^{6}, \gamma_{\min }=10^{4}$. We then adjust $\rho$ to the best fitting value of $\rho=510^{8} \mathrm{~cm}$. The result is shown in Fig. 1, which closely fits observations of NGC 5548 in the $0.2-10 \mathrm{keV}$ band.

In our simulation, we have used a one power-law model. If we assume electrons having a power law energy distribution, we arrive at a soft X-ray excess spectrum with no need to add in any other mechanism or component. This is much simpler than other models, in which at least one another mechanism or component is needed, either a thermal component such as an accretion disk, bremsstrahlung, or a black body, or a second nonthermal component. We think its greater simplicity may make this mechanism appealing in applications such as soft X-ray excess. Furthermore, we have only one variable $\rho$. From the spectrum's shape or its indexes in two bands, we can find the index of the electrons' energy distribution. If we give $B$ a typical value as before, from the spectrum's turnoff point, we can find $\gamma_{\mathrm{c}}, \gamma_{\max }, \gamma_{\min }$ and $\rho$. We can get 
almost all the information from the spectrum itself without requiring other variables. The number of parameters in this model is far less than in any other that we have seen. This mechanism also gives us the magnitude of $\rho$, which is not the case using other models.

We can summarise the advantages of the synchrocurvature method as follows:

1) it gives a curved spectrum without adding in any other component;

2 ) it uses only one variable $\rho$, and the other parameters can be obtained from the spectrum itself;

3 ) it gives new information, the value of $\rho$, which is useful in considering a more refined magnetic structure.

We note the following considerations: we use only one component, generated by one electron population in both the soft and hard X-ray band, then we would expect the soft and hard X-ray emission to vary simultaneously, and the variability amplitude to be similar. Recent observations proposed that no time lag is found between the soft and hard X-ray band in other sources (Edelson et al. 2000; Nandra et al. 2000), indicating that a single component dominates the emission over the whole X-ray band. This contradicts previous observations of NGC 5548, but may be evidence as to the applicability of our mechanism. More observations and theoretical work are needed to clarify this point. We suggest that synchro-curvature is just one of the main physical process, together with other physical process such as soft X-ray reprocessing, which explains the variable part of the optical/UV emissions.

Also the magnetic field is not constrained at $1 \mathrm{G}$. If it is $1 \mathrm{G}$, the scale of its curvature is $510^{8} \mathrm{~cm}$ and is about $10^{-5}$ times the scale of the dimension of the system. If we use $B \sim 1 \mathrm{mG}$, the scale of the magnetic field would be comparable to the system. Thus, we also need a more refined value of the magnetic field intensity to allow a more detailed application of this model. In order to confidently apply such a model to this problem, more attention needs to be paid to the details of the magnetic field in AGNs.

\section{Conclusions}

In this paper, we present a synchro-curvature mechanism to model the spectrum of a well-observed source, NGC 5548, and find that the model closely fits the observed data.

We propose that a synchro-curvature mechanism may be applicable to analyse the soft X-ray excess in AGNs and related problems. However, more attention needs to be paid to the details of this mechanism. Future observations and theoretical work proving the same origin of the soft and hard X-ray (at least the main emissions or the original emissions of them) are crucial to the application of this newly-proposed mechanism. If this model proves to be applicable, more information is required about the magnetic fields in AGNs.

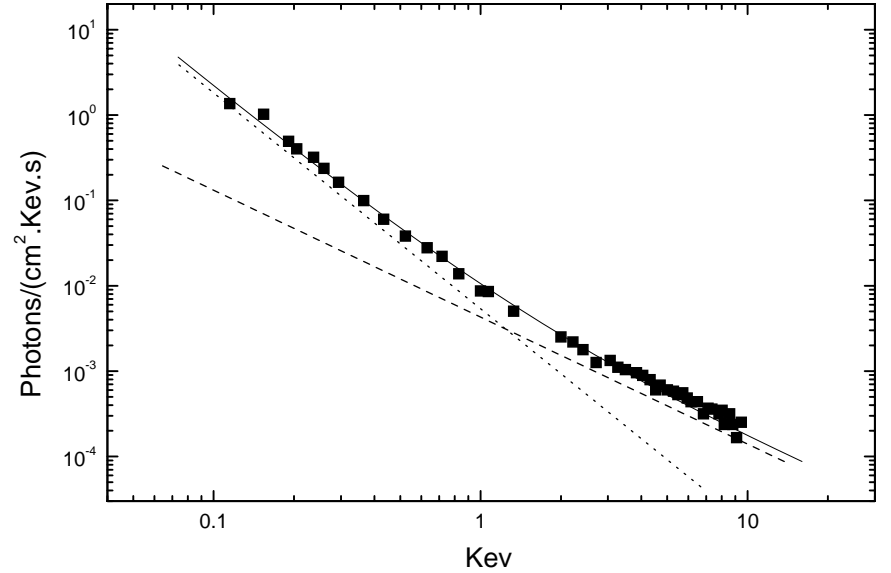

Fig. 1. Very good fitting (solid line) of the synchro-curvature model to observations (squares) of NGC 5548 in the $0.2-10 \mathrm{keV}$ band. For comparison, the dotted line and long-dashed line shows the synchrotron and curvature spectrum emitted by the same electron distribution, respectively. In the model, if the intensity of the magnetic field is $1 \mathrm{G}$, its curvature radius would be $510^{8} \mathrm{~cm}$ for best fitting

Acknowledgements. It is a pleasure to thank Dr. A. Laor and J. M. Yang for their useful suggestions. This work was supported by the National Natural Science Foundation and the National "Climbing Project" of China.

\section{References}

Arnaud, K. A., Branduardi-Raymont, G., Culhane, J. L., et al. 1985, MNRAS, 217, 105

Barcons, X., \& Fabian, A. C. (eds.) 1992, The X-ray Background (Cambridge Univ. Press, Cambridge)

Cheng, K. S., \& Zhang, J. L. 1996, ApJ, 463, 271

Czerny, B., \& Elvis, M. 1987, ApJ, 321, 305

Done, C., \& Krolik, J. H. 1995, ApJ, 463, 144

Edelson, R., Koratkar, A., Nandra, K., et al. 2000, ApJ, 534 180

Elvis, M., Maccacaro, T., Wilson, A. S., et al. 1978, MNRAS, 183,129

Elvis, M., Wilkes, B. J., \& Tanabaum, H. 1985, ApJ, 292, 367

George, I. M., Turner, T. J., Hagai Netzer., et al. 1998, ApJS, 114,73

Laor, A., Fore, F., Elvis, M., et al. 1997, ApJ, 477, 93

Marshall, N., Warwick, R. S., \& Pounds, K. A. 1981, MNRAS, 194, 987

Mushotzky, R. F. 1984, Adv. Space Res., 3, 517

Nandra, K., Le, T., George, I. M., et al. 2000, ApJ, 544, 734

Pravdo, S. H., \& Marshall, F. E. 1984, ApJ, 281, 504

Rush, B., \& Malkan, M. A. 1996, ApJ, 456, 466

Turner, T. J., \& Pounds, K. A. 1989, MNRAS, 240, 883

Turner, T. J., George, I. M., \& Mushotzky, R. F. 1993, ApJ, 412,72

Walter, R., \& Fink, H. H. 1993, A\&A, 274, 105

Walter, R., Orr, A., Courvoisier, T. J.-L., et al. 1994, A\&A, 285, 119

Zhang, J. L., \& Cheng, K. S. 1995, Phys. Lett. A, 208, 47

Zhang, J. L., Xia, T. S., \& Yang, J. M. 2000, Phys. Lett. A, 275,315

Zheng, W., Kriss, G. A., Telfer, R. C., et al. 1997, ApJ, 475, 469 